\title{
An Unusual Cause of Seizure in Clinical Practice
}

\author{
A HOSSAIN ${ }^{1}$, QT ISLAM ${ }^{2}$, MMR SIDDI QUI $^{3}$, YU RAHMAN ${ }^{4}$, RA SHIPA $^{5}$
}

\begin{abstract}
:
Neurocysticercosis (NCC), the infection caused by the larval stage of the tapeworm Taenia solium, is the most common parasitic disease of the nervous system (NS) in humans, where it is the leading cause of late-onset seizures in the endemic areas. Fortunately it is still rare in Bangladesh due to some religious binding in eating pork. Here we present a young man who came to us with Neurocysticercosis as the cause of recurrent seizure and various neurological manifestations.
\end{abstract}

Keyword: Neurocysticercosis, Taenia solium, seizure.

\section{Introduction:}

Neurocysticercosis (NCC), the infection caused by the larval stage of the tapeworm Taenia solium, is the most common parasitic disease of the nervous system (NS) in humans. ${ }^{1}$ It affects millions of people worldwide with a prevalence of $3 \%$ to $6 \%$ in developing countries, where it is the leading cause of late-onset seizures in the endemic areas. ${ }^{2}$ Endemic areas include Mexico and Latin America, sub-Saharan Africa, India, and East Asia. Fortunately it is still rare in Bangladesh due to some religious binding in eating pork. During the last two decades, the introduction of computed tomography (CT) and later magnetic resonance imaging (MRI) permitted the identification of this previously under diagnosed disease in our country. ${ }^{1}$ Here we reported a case of NCC diagnosed on the basis of clinical context and brain imaging.

\section{Case Report:}

A 26-year-old Hindu young man from Tangail admitted in the medicine unit III of Dhaka Medical College Hospital with the complaints of recurrent seizure for last 2 months, headache for 20 days, irrelevant talk and emotional liability for last 7 days. He had no history of fever, cough, neck stiffness, but he complained of double vision for last few days. On query he gave history of taking pork meat in various occasions. His past medical history was unremarkable. Clinical examination revealed that he had emotional liability, bilateral $6^{\text {th }}$ cranial nerve palsy, right sided upper motor type $7^{\text {th }}$ cranial nerve palsy, bilateral papilloedema and bilateral extensor planter reflexes. All other clinical examination was unremarkable.

Investigations revealed total white blood cell count 10,000/ $\mathrm{mm}^{3}$ eosinophil count $10 \%$, on cerebro spinal fluid (CSF) examination total cell count $5 / \mathrm{mm}^{3}$, all were lymphocyte, protein- $60 \mathrm{mg} / \mathrm{dl}$ and all other biochemical reports were normal. Then we went for MRI of the brain. On T2 weighted and FLAIR images, which showed a well-defined, thin, hyperintense capsule with marked perifocal edema in the left parietal lobe. The center of the lesion was hypointense. There was also marked focal oedema involving right parietal region (fig-1). Corresponding postcontrast axial T1-weighted MRI showed multiple very small enhanced nodular tissue lesions involving bilateral parietal, temporal and frontal region. Some of them showed ring enhancement (fig-2). From the clinical context and MRI findings we diagnosed him as a case of Neurocysticercosis, though we did not go for invasive brain biopsy and serological test was not available. We gave him tablet albendazole $400 \mathrm{mg}$ per oral 2 times daily for 2 weeks, tablet prednisolone $40 \mathrm{mg}$ per oral daily for 2 weeks then gradual tapering. After 4 weeks he came to us for follow up and it was surprising to see that he had no seizure, headache or double vision. All neurological abnormal findings resolved and he had no papilloedema. Follow up MRI after 1 month revealed no focal brain oedema and all the enhanced nodular lesions disappeared (fig.-3,4). Finally it was confirmed a case of recurrent seizure due to Neurocysticercosis.

1. Assistant Professor, Dept. of Medicine, Dhaka Medical College, Dhaka,

2. Quazi Tarikul Islam, Professor, Dept. of Medicine, Dhaka Medical College, Dhaka,

3. FCPS(Med) P-II Course student, Dept. of Medicine, Dhaka Medical College, Dhaka,

4. Assistant Registrar, Dept. of Medicine, Dhaka Medical College, Dhaka,

5. Postgraduate resident, Dept. of Medicine, Dhaka Medical College, Dhaka,

Correspondence: Dr. Ahmed Hossain, Assistant Professor, Dept. of Medicine, Dhaka Medical College, Dhaka. E-mail:dr.mahmud 99@yahoo.com 


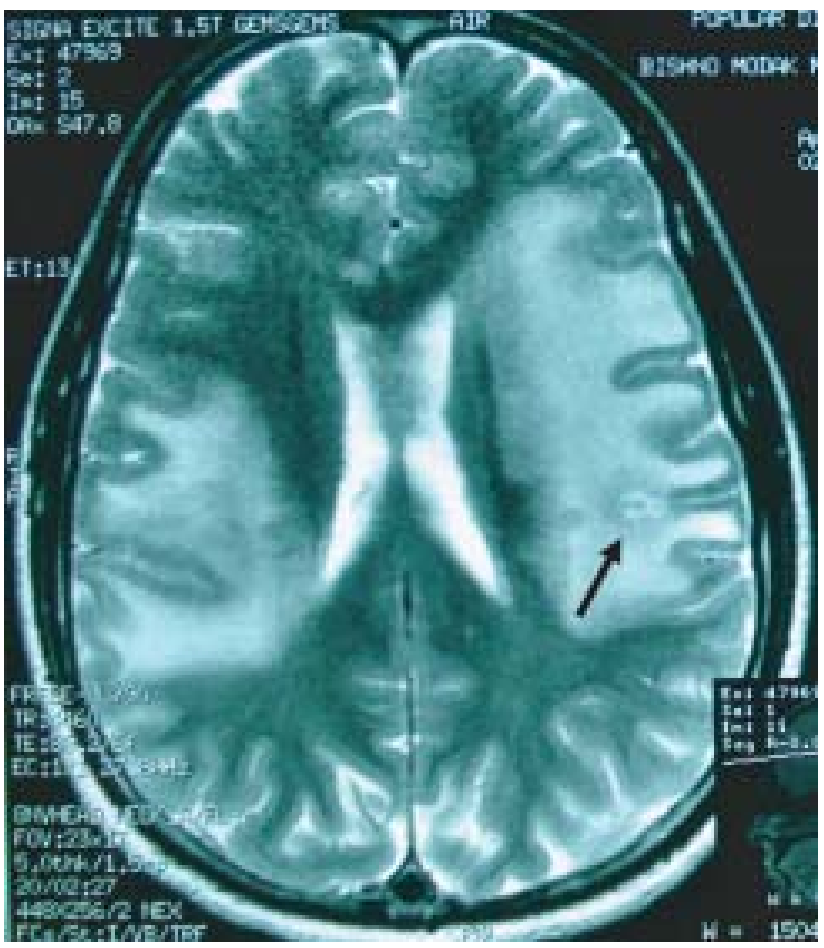

Fig.-1: T2 weighted axial MRI of the brain shows a welldefined, thin, hyperintense capsule with hypointense center (arrow) with marked perifocal edema in the left parietal lobe \& also in right parietal lobe.

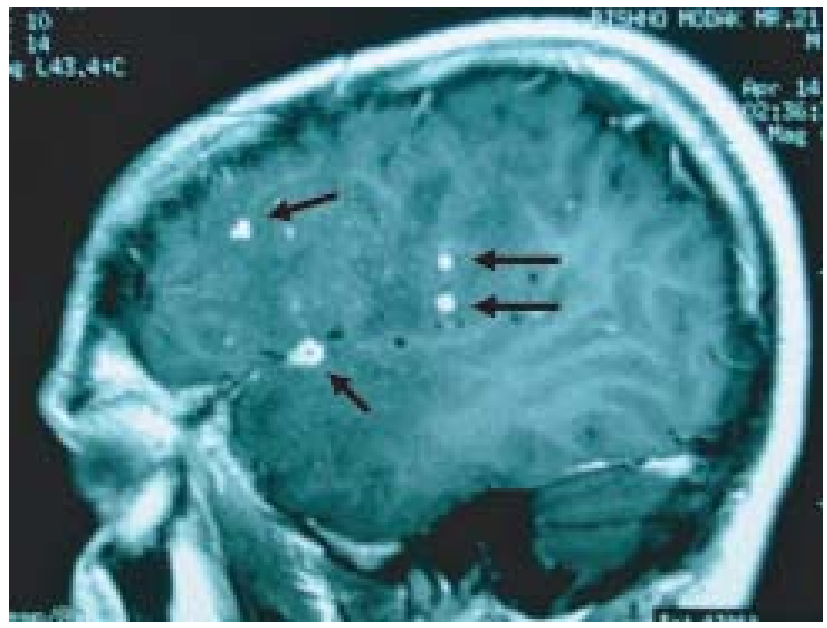

Fig.-2: Post contrast T1-weighted MRI showed multiple very small enhanced nodular tissue lesions (arrows) involving parietal, temporal and frontal lobes with perifocal oedema.

\section{Discussion:}

Humans are definitive hosts and pigs are the intermediate host for Taenia solium (TS). ${ }^{1,2}$ Typically, people develop taeniasis after eating pork containing encysted larvae, which develop into tapeworms in the small bowel. ${ }^{1}$ Human can also be intermediate host of TS by ingesting

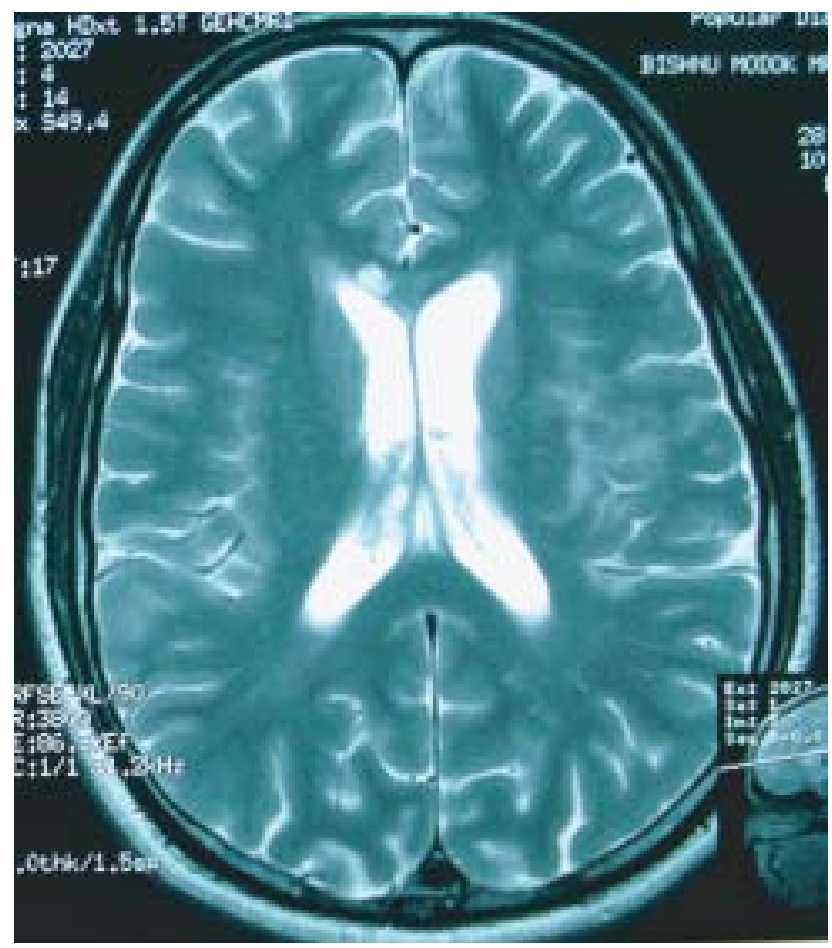

Fig.-3: Follow up T2 weighted axial MRI of the brain shows marked improvement from previous one.

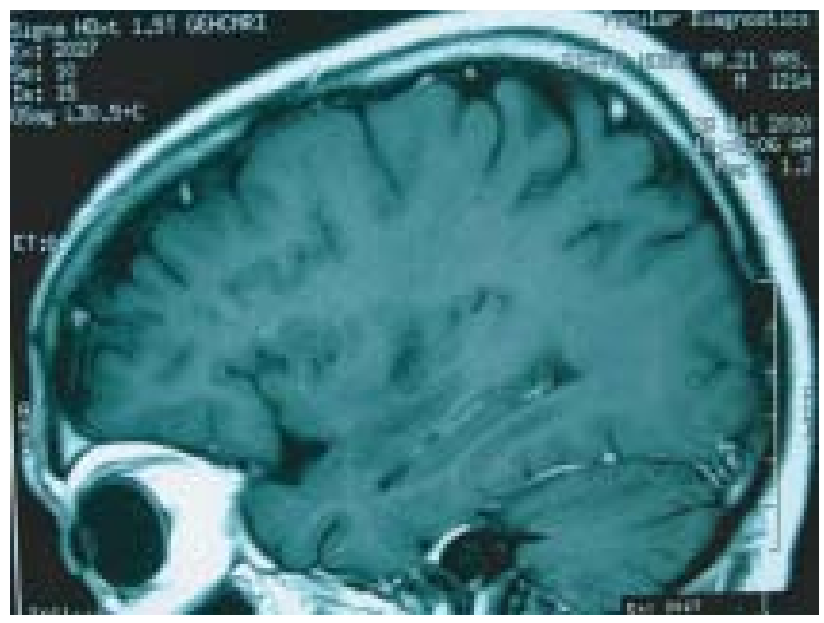

Fig.-4: Follow up post contrast $T 1$ weighted MRI showing disappearance of all enhanced nodular tissue lesions.

ova which mature into larvae, penetrate bowel walls and migrate hematogenously into central nervous system (CNS), eye, muscle and skin. ${ }^{1,3}$ Consumption of semi- or improperly cooked pork, contaminated salad, and inadequate hygiene are the main cause of infection. ${ }^{3}$ In Bangladesh, more than $90 \%$ peoples are Muslim who 
have religious binding not to eat pork and thats why cysticercosis is very rare.

Most NC are asymptomatic, common manifestations in descending order are seizures (70\% to $90 \%$ of acutely symptomatic patients), headache, learning disability, behavior changes, psychomotor involution and increased intracranial pressure. ${ }^{4}$ Headache usually indicates the presence of hydrocephalus, meningitis or increased intracranial pressure (ICP). ${ }^{4}$ Patients with NCC usually have partial seizures with or without secondary generalization. ${ }^{1}$ At the time of presentation, most patients have an active cyst in the form of vesicular or colloidal stage, which become calcified granular in chronic cases. ${ }^{4,5}$ It causes seizure by several factors: inflammation, gliosis, raised ICP and predilection for the cysts to involve the frontal and temporal lobes. ${ }^{4,5}$

The diagnosis of NCC is difficult because clinical manifestations are nonspecific, most neuroimaging findings are not pathognomonic, and some serologic tests have low sensitivity and specificity. CT and MRI findings depend on staging of NC. ${ }^{6}$ In the early stage, larva invasion appears as localized edema on T2-weighted images and displays nodular tissue enhancement. Then cyst encircles the scolex in the vesicular stage. The scolex is usually visualized as a pathognomonic 'hole-with-dot' lesion and numerous NCC resemble 'Swiss cheese' appearances. Most of them show enhancing ring lesions with perifocal edema on CT and MRI. ${ }^{7}$ But the definite diagnosis depends on finding a TS larva from CNS studies, including: biopsy, a scolex within cyst on neuroimaging, or subretinal parasites via fundoscopy. ${ }^{2}$

Treatment options include antiparasitic drugs, surgery and symptomatic medication. Albendazole $(15 \mathrm{mg} / \mathrm{kg} /$ day per oral 2 divided doses for 8-30 days; not to exceed $800 \mathrm{mg} / \mathrm{d}$ ) and praziquantel (50 mg/kg/day per oral 3 divided doses for 15 days) are the principal antiparasitic drugs used to treat NCC. ${ }^{8}$ Seizures secondary to NCC usually respond well to first-line antiepileptic drugs. ${ }^{1}$ Corticosteroids are frequently used to decrease neurological symptoms due to the death of the parasite and are the primary management for chronic cysticercosis arachnoiditis or encephalitis. ${ }^{1}$ Follow-up imaging study is recommended after 2-3 months, especially in cases in which anticysticercal medications are used as a diagnostic tool.

\section{Conflict of Interest : None}

\section{References:}

1. García HH, Evans CAW, Nash TE et al. Current Consensus Guidelines for Treatment of Neurocysticercosis. Clinical Microbiology Reviews 2002;15(4):747-56

2. Chang HY, Yang CM, Cheng TC et al. Neurocysticercosis: a case report. Chin J Radiol 2009; 34: 201-05

3. Hoberg EP. Taenia tapeworms: their biology, evolution and socioeconomic significance. Microbes Infect 2002; 4: 859-66

4. DeGiorgio CM, Medina MT, Duron R et al. Neurocysticercosis. Epilepsy Currents 2004; 4(3): 107-11

5. Del Brutto OH, Santibanez R, Noboa CA et al. Epilepsy due to neurocysticercosis: Analysis of 203 patients. Neurology 1992;42:389-92

6. Rocca U, Rosell A, Alvarez C. Surgical options in neurocysticercosis therapy. Neurosurg Q 2005; 15: 5-13

7. Garcýa HH, Brutto OH. Review article: Imaging findings in neurocysticercosis. Acta Tropica 2003; 87: 71-8

8. Garg RK. Medical management of neurocysticercosis. Neurol India 2001;49(4):329-37 